\title{
Damage Description with Related Crack Initiation and Propagation Conditions
}

\author{
Z. Mróz and A. Seweryn* \\ Institute of Fundamental Technological Research, Swietokrzyska 21,00-049 Warsaw, Poland \\ * Bialystok University of Technology, Faculty of Mechanics, Wiejska 45c, 15-351 Bialystok, Poland
}

\begin{abstract}
The damage accumulation condition expressed in terms of traction components on a physical plane is considered for both monotonic and cyclic loading conditions. The crack initiation is assumed to correspond to a critical value of damage parameter on a maximum damage plane. A non-local condition is formulated for singular stress or strain regimes. The model is applied to predict damage distribution within the element for cyclic loading condition; in particular for combined torsion and bending. The damage tensors are introduced to describe the predicted damage distribution.
\end{abstract}

\section{NTRODUCTION}

The present paper is concerned with the description of damage evolution for brittle materials such as rock, concrete, ceramics, some glassy polymers, and for metals under variable loading inducing fatigue crack initiation and propagation within the nominally elastic regime. The macroscopic plastic deformation can then be neglected and the inelastic strain is associated with the microcracking process. For high-cycle fatigue, the microplastic effects occur at the dislocation level and the macroscopic response is usually treated as elastic with negligible effect of microcracks on the effective elastic moduli.

In formulating constitutive models the damage is usually described by scalar or tensor state variables representing average crack density and orientation within the macroscopic element. The evolution rules for damage and compliance are then formulated. Starting from the early Kachanov work [1] who defined the scalar measure of damage, the subsequent investigators used different measures, $\mathrm{cf}$. Lemaitre [2], Dragon and Mróz [3], Krajcinovic [4], Murakami [5], Onat and Leckie [6], Simo and Ju [7], Chaboche [8] and others. The anisotropic crack or fabric distribution by various order tersors was thoroughly discussed by Onat and Leckie [6], Kanatani [9], and recently by Lubarda and Krajcinovic [10].

In the present work, the damage distribution within the material element will be described by providing the rule of distribution of a scalar damage variable $\omega_{n}(\underline{n})$ on planes of varying orientation $\underline{n}$. The macrocrack initiation is assumed to occur along the plane of maximal damage value. For the specified distribution of damage the respective tensor measure of crack density distribution can be identified. The elastic compliance variation due to damage can be neglected for multiaxial fatigue problems. However, when needed, this variation can be calculated by introducing the interfacial strain components due to damage and averaging them over all plane orientations.

\section{NON-LOCAL STRESS OR STRAIN CONDITION OF BRITTLE FAILURE}

To provide uniform treatment of crack initiation and propagation from singular and regular stress concentrations, the non-local stress condition was proposed by Seweryn and Mróz [12] and applied to predict both critical load value for crack initiation and also orientation of crack propagation. The fatigue 


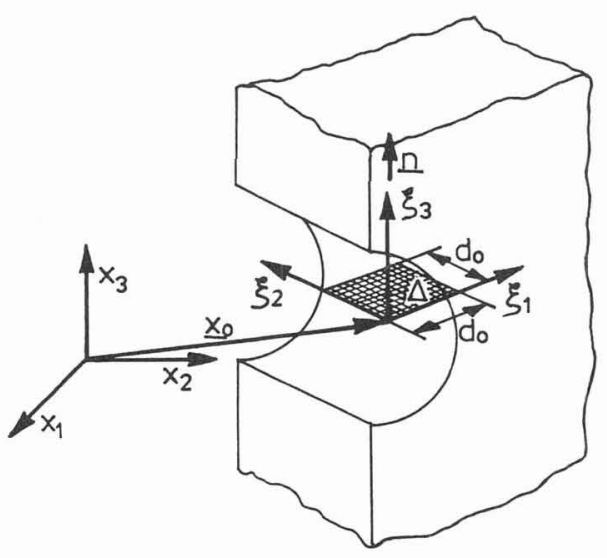

Figure 1. Physical plane $\Delta$ associated with the local coordinate system $\left(\xi_{1}, \xi_{2}, \xi_{3}\right)$ and the global system $\left(x_{1}, x_{2}, x_{3}\right)$.

damage accumulation and crack propagation was studied using this conditions by Seweryn and Mróz [13,14].

Consider a local plane $\Delta$ specified by a position vector $\underline{x}_{o}$ and a unit normal vector $\underline{\underline{n}}$, Figure 1 . The traction vector $\underline{t}=\underline{\sigma} \underline{n}$ aciting on the physical plane can be expressed in the local coordinate system by

$$
\underline{\Sigma}\left(\tau_{\mathrm{n} 1}, \tau_{\mathrm{n} 2}, \sigma_{\mathrm{n}}\right)=\underline{\mathrm{Q}} \underline{\mathrm{t}}=\underline{\mathrm{Q}} \underline{\sigma} \underline{\mathrm{n}}
$$

where $\underline{Q}$ is an orthogonal tensor of transformation from the global system $\left(\mathrm{x}_{1}, \mathrm{x}_{2}, \mathrm{x}_{3}\right)$ to the local system $\left(\xi_{1}, \xi_{2}, \xi_{3}\right)$ with $\xi_{3}$ following the normal to the plane. The shear stresses within the plane follow coordinate axes $\xi_{1}$ and $\xi_{2}$ and the resultant shear stress equals

$$
\tau_{\mathrm{n}}=\left(\tau_{\mathrm{n} 1}^{2}+\tau_{\mathrm{n} 2}^{2}\right)^{1 / 2}
$$

The local stress condition of brittle failure is formulated in terms of the damage stress function on any physical plane, thus the crack initiation condition is

$$
\mathbf{R}_{\mathrm{f \sigma}}=\max _{\left(\underline{\underline{n}}, \underline{x}_{0}\right)} R_{\sigma}\left(\sigma_{\mathrm{n}} / \sigma_{\mathrm{c}}, \tau_{\mathrm{n}} / \tau_{\mathrm{c}}\right)=1
$$

a)

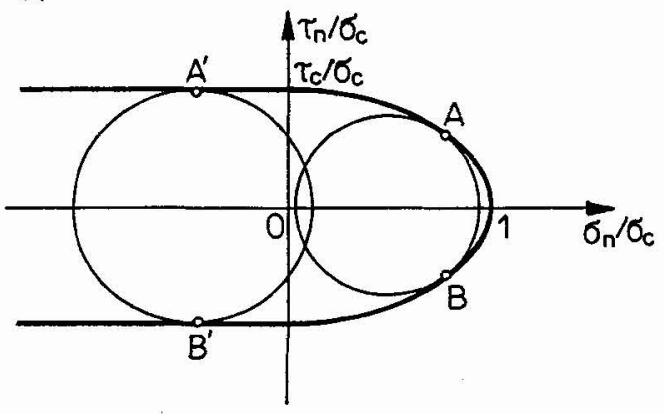

b)

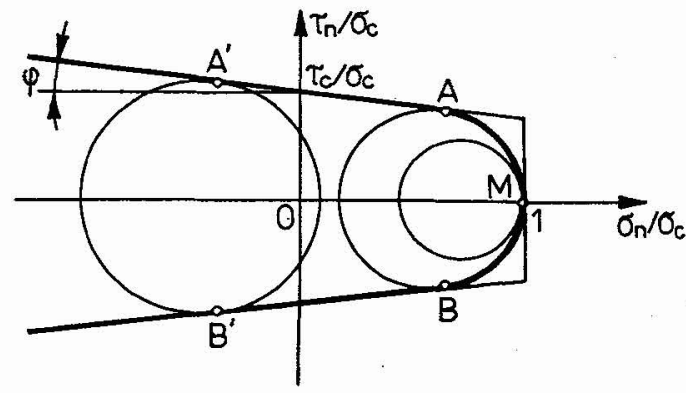

Figure 2. Stress failure functions: a) elliptical condition combined with the maximum shear condition, b) Coulomb condition combined with limit tensile strenght condition. where $0 \leq R_{\text {fo }} \leq 1$ is the brittle failure factor, $\sigma_{c}$ and $\tau_{c}$ denote the critical values of normal and shear stresses. The damage stress function is expressed in terms of contact stress components $\sigma_{\mathrm{n}}$ and $\tau_{\mathrm{n}}$. Let us note that besides the crack initiation condition, we may specify also the variation of $R_{\sigma}$ with respect to plane orientation and assume that damage distribution follows the variation of $R_{\sigma}$.

In the previous study [12], the elliptic condition combined with the shear condition was applied, thus

$$
\mathbf{R}_{\sigma}=\left[\left(\frac{\left\langle\sigma_{n}\right\rangle}{\sigma_{c}}\right)^{2}+\left(\frac{\tau_{n}}{\tau_{c}}\right)^{2}\right]^{1 / 2}
$$

where $\left\langle\sigma_{n}\right\rangle=\sigma_{n}$ for $\sigma_{n}>0$ and $\left\langle\sigma_{n}\right\rangle=0$ for $\sigma_{\mathrm{n}}<0$. An alternative condition is provided by the Coulomb condition combined with the normal stress condition, thus

$$
\mathbf{R}_{\sigma}=\min \left\{\frac{1}{\tau_{c}}\left(\left|\tau_{n}\right|+\sigma_{n} \tan \varphi\right), \frac{\left\langle\sigma_{n}\right\rangle}{\sigma_{c}}\right\}
$$

Figures $2 \mathrm{a}, \mathrm{b}$ present these two conditions in the stress plane. 
For singular or quasi-singular stress distributions with very large stress gradients, the failure condition is averaged over the area $d_{0} \times d_{0}$, thus

$$
\overline{\mathbf{R}}_{f \sigma}=\max _{\left(\underline{\underline{n}, \underline{x}_{0}}\right)} \bar{R}_{\sigma}\left(\frac{\sigma_{\mathrm{n}}}{\sigma_{\mathrm{c}}}, \frac{\tau_{\mathrm{n}}}{\tau_{\mathrm{c}}}\right)=\max _{\left(\underline{\mathrm{n}}, \underline{\underline{x}}_{0}\right.}\left[\frac{1}{\mathrm{~d}_{0}^{2}} \int_{0}^{\mathrm{d}_{0} \mathrm{~d}_{0}} \int_{0}^{\mathrm{R}} \mathrm{R}_{\sigma}\left(\frac{\sigma_{\mathrm{n}}}{\sigma_{\mathrm{c}}}, \frac{\tau_{\mathrm{n}}}{\tau_{\mathrm{c}}}\right) \mathrm{d} \xi_{1} \mathrm{~d} \xi_{2}\right]=1
$$

where $d_{0}$ specifies the non-locality domain. The value of $d_{0}$ can be related to microstructural parameters, for instance, grain size. To make the non-local model equivalent to Griffith-Irwin crack propagation condition $\mathrm{K}_{\mathrm{I}}=\mathrm{K}_{\mathrm{Ic}}$ in Mode $\mathrm{I}$, the value of $\mathrm{d}_{0}$ can be identified as [11]

$$
\mathrm{d}_{\mathrm{o}}=\frac{1}{2 \pi}\left(\frac{2 \mathrm{~K}_{\mathrm{Ic}}}{\sigma_{\mathrm{c}}}\right)^{2}
$$

where $\mathrm{K}_{\mathrm{Ic}}$ is the critical value of the stress intensity factor.

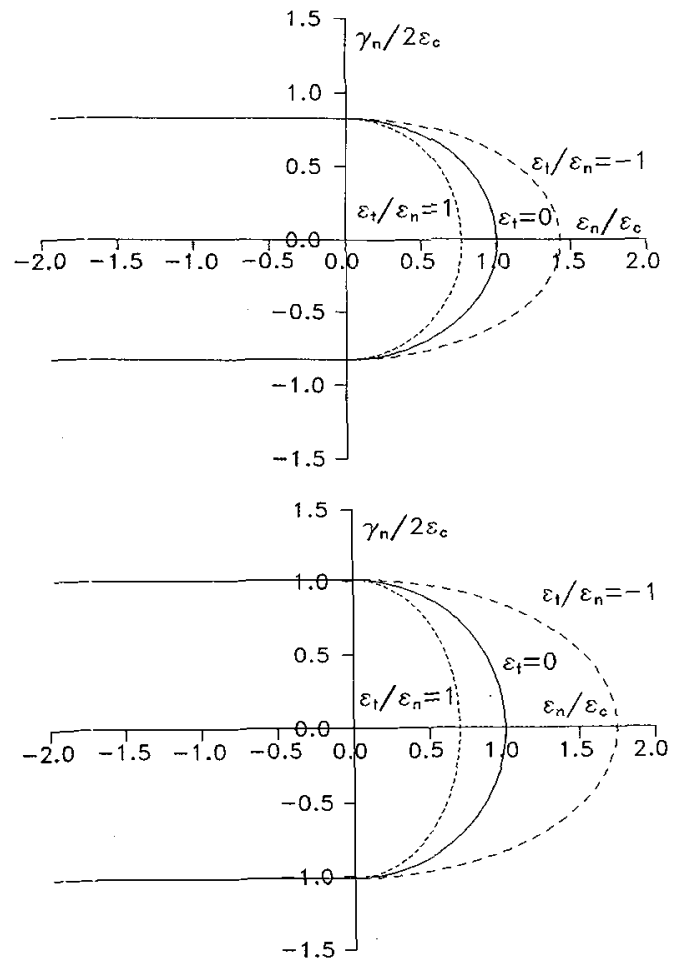

Figure 3. Stress failure function (4) in the strain plane for $\tau_{\mathrm{c}} / \sigma_{\mathrm{c}}=1 / \sqrt{3}, v=0.3$ : a) plane stress, b) plane strain state.
The damage accumulation and temperature effect can be accounted for by assuming that

$$
\begin{aligned}
& \sigma_{\mathrm{c}}=\sigma_{\mathrm{c}}\left(\mathrm{T}, \omega_{\mathrm{n} \sigma}\right)=\sigma_{\mathrm{co}}(\mathrm{T})\left(1-\omega_{\mathrm{n} \sigma}\right)^{\mathrm{p}} \\
& \tau_{\mathrm{c}}=\tau_{\mathrm{c}}\left(\mathrm{T}, \omega_{\mathrm{n} \sigma}\right)=\tau_{\mathrm{co}}(\mathrm{T})\left(1-\omega_{\mathrm{n} \sigma}\right)^{\mathrm{p}}
\end{aligned}
$$

where $\sigma_{c o}$ and $\tau_{c o}$ are the failure stresses for the undamaged material.

An alternative condition for failure can be expressed in terms of contact strain components $\gamma_{\mathrm{n} 1}$, $\gamma_{\mathrm{n} 2}, \varepsilon_{\mathrm{n}}$ on the plane $\Delta$. Introducing the resultant shear stress $\gamma_{\mathrm{n}}=\left[\gamma_{\mathrm{nl}}{ }^{2}+\gamma_{\mathrm{n} 2}{ }^{2}\right]^{1 / 2}$, the local failure condition can be expressed as follows

$$
\mathrm{R}_{\mathrm{f} \varepsilon}=\max _{\left(\underline{\underline{n}}, \underline{x}_{\mathrm{o}}\right)} \mathrm{R}_{\varepsilon}\left(\frac{\varepsilon_{\mathrm{n}}}{\varepsilon_{\mathrm{c}}}, \frac{\gamma_{\mathrm{n}}}{\gamma_{\mathrm{c}}}\right)=1
$$

where $\varepsilon_{\mathrm{c}}, \gamma_{\mathrm{c}}$ are the critical failure strain values and $\mathrm{R}_{\varepsilon}$ is the strain failure function. Similarly to (4), we can postulate

$$
\mathrm{R}_{\varepsilon}=\left[\left(\frac{\left\langle\varepsilon_{\mathrm{n}}\right\rangle}{\varepsilon_{\mathrm{c}}}\right)^{2}+\left(\frac{\gamma_{\mathrm{n}}}{\gamma_{\mathrm{c}}}\right)^{2}\right]^{1 / 2}
$$

The non-local strain failure condition can be formulated analogously to (6), thus

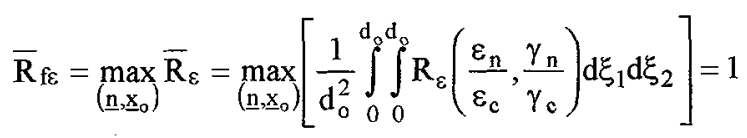

It should be noted that the stress and strain conditions are not equivalent since $\varepsilon_{\mathrm{n}}$ depends on $\sigma_{\mathrm{n}}$ and $\sigma_{\mathrm{tl}}$, $\sigma_{\mathrm{t} 2}$ acting within the contact plane and $\sigma_{\mathrm{n}}$ depends on strain components $\varepsilon_{\mathrm{n}}, \varepsilon_{\mathrm{t} 1}, \varepsilon_{\mathrm{t} 2}$. Figures $3 \mathrm{a}, \mathrm{b}$ present the stress failure condition (4) in the strain plane for plane stress and plane strain state. It is seen that the elliptical condition is affected by the ratio $\varepsilon_{\mathrm{t}} / \varepsilon_{\mathrm{n}}$. 


\section{MULTIAXIAL FATIGUE LOADING}

Let us now discuss the damage evolution rule in the case of multiaxial fatigue loading within the elastic domain so the macroplastic strains do not occur. The growth of brittle damage is now governed by the relation

$$
\mathrm{d} \omega_{n \sigma}=\mathrm{d} \omega_{n \sigma}\left(\underline{\Sigma}, \underline{\mathrm{d} \Sigma}, \omega_{n \sigma}\right)
$$

The domain of no damage accumulation is specified by the inequality

$$
\mathbf{R}_{\sigma o}\left(\frac{\sigma_{\mathrm{n}}}{\sigma_{\mathrm{o}}}, \frac{\tau_{\mathrm{n}}}{\tau_{\mathrm{o}}}\right)<1
$$

and the damage initiation locus corresponds to the value $R_{f \sigma o}=R_{\sigma o}=1$. Here $\sigma_{o}$ and $\tau_{0}$ are the damage initiation stresses in tension and shear. For large values of stress gradient the non-local condition is expressed as follows

$$
\overline{\mathbf{R}}_{\text {foo }}=\max _{\left(\underline{\underline{n}}, \underline{\underline{x}}_{0}\right)} \overline{\mathbf{R}}_{\sigma o}=\max _{\left(\underline{n}, \underline{x}_{0}\right)}\left[\frac{1}{\mathrm{~d}_{0}^{2}} \int_{0}^{\mathrm{d}_{0} \mathrm{~d}_{0}} \int_{0}^{\mathrm{R}} \mathrm{R}_{\sigma o}\left(\frac{\sigma_{\mathrm{n}}}{\sigma_{0}}, \frac{\tau_{\mathrm{n}}}{\tau_{0}}\right) \mathrm{d} \xi_{1} \mathrm{~d} \xi_{2}\right]=1
$$

Consider the domain $\Omega^{*}$ bounded by damage initiation and stress failure curves, Figure 4 . Assume, for simplicity that $\sigma_{o}=f \sigma_{c}, \tau_{o}=f \tau_{c}$. Consider a family of curves

a)

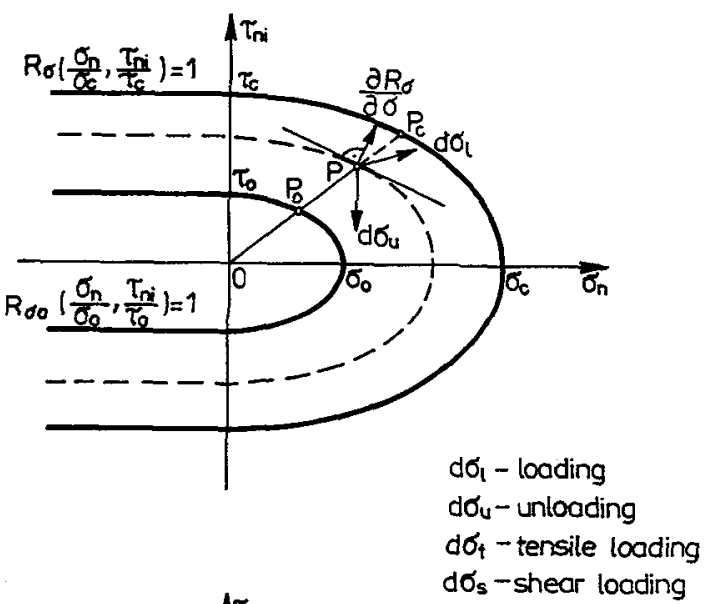

$$
R_{\sigma}=\left[\left(\frac{\left\langle\sigma_{n}\right\rangle}{\sigma_{c}}\right)^{2}+\left(\frac{\tau_{n}}{\tau_{c}}\right)^{2}\right]^{1 / 2}=\text { const. }
$$

For $\mathbf{R}_{\sigma}=1$, the curve coincides with the stress failure locus, for $R_{\sigma}=f$, with the damage initiation locus $\mathbf{R}_{\sigma o}=1$. The damage growth is specified by the following relation

$$
\mathrm{d} \omega_{\mathrm{n} \sigma}=\Psi_{\sigma}\left(\mathrm{R}_{\sigma}\right) \mathrm{d} \hat{\mathrm{R}}_{\sigma}
$$

where the damage accumulation function $\Psi_{\sigma}$ can be assumed in the form

$$
\begin{aligned}
& \Psi_{\sigma}\left(R_{\sigma}\right)=A_{\sigma}\left(\frac{R_{\sigma}-R_{\sigma o c}}{1-R_{\sigma o c}}\right)^{\mathbf{n}_{\sigma}} \frac{1}{1-R_{\sigma o c}} \\
& \mathbf{R}_{\sigma o c}=R_{\sigma} / R_{\sigma o}
\end{aligned}
$$

The material parameters $n_{\sigma}$ and $A_{\sigma}$ are specified from experimental data. The increment $d \hat{R}_{\sigma}$ is specified by the formula

$$
d \hat{R}_{\sigma}=\left\{\begin{array}{lll}
d R_{\sigma} & \text { for } d R_{\sigma} \geq 0 \text { and } R_{\sigma} \geq f \\
0 & \text { for } d R_{\sigma}<0 \text { or } R_{\sigma}<f
\end{array}\right.
$$

and

$$
\mathrm{dR}_{\sigma}=\frac{\partial \mathbf{R}_{\sigma}}{\partial \sigma_{\mathrm{n}}} \mathrm{d} \sigma_{\mathrm{n}}+\frac{\partial \mathbf{R}_{\sigma}}{\partial \tau_{\mathrm{nl}}} \mathrm{d} \tau_{\mathrm{n}}+\frac{\partial \mathbf{R}_{\sigma}}{\partial \omega_{\mathrm{n} \sigma}} \mathrm{d} \omega_{\mathrm{n} \sigma}
$$

Neglecting the effect of damage accumulation

Figure 4. The brittle failure function and the microplastic damage initiation function. The damage accumulation occurs due to: a) variation of the failure function, b) variation of the stress state

on function $R_{\sigma}$, for the damage condition (4) we have 


$$
\mathrm{dR}_{\sigma}=\frac{1}{\mathrm{R}_{\sigma}}\left[\frac{\left\langle\sigma_{\mathrm{n}}\right\rangle \mathrm{d} \sigma_{\mathrm{n}}}{\sigma_{\mathrm{c}}{ }^{2}}+\frac{\tau_{\mathrm{n}} \mathrm{d} \tau_{\mathrm{n}}}{\tau_{\mathrm{c}}{ }^{2}}\right]
$$

An alternative specification of loading-unloading domains can be proposed by introducing two planes moving with stress point [13]

$$
\begin{aligned}
& \phi_{1}=\hat{\sigma}_{\mathrm{n}}-\sigma_{\mathrm{n}}^{+}(\mathrm{t})=0 \\
& \phi_{2}=\hat{\tau}_{\mathrm{n}}{ }^{2}-\tau_{\mathrm{n}}{ }^{2}(\mathrm{t})=0
\end{aligned}
$$

where $\sigma_{n}^{+}(t)$ and $\tau_{n}(t)$ are the actual values of tensile and shear stresses and damage growth is assumed to occul when

$$
\begin{aligned}
& \phi_{1}=0, \quad \mathrm{~d} \phi_{1}>0, \quad \text { or } \quad \hat{\sigma}_{\mathrm{n}}=\sigma_{\mathrm{n}}^{+}(\mathrm{t}), \quad \mathrm{d} \hat{\sigma}_{\mathrm{n}}=\mathrm{d} \sigma_{\mathrm{n}}>0 \\
& \phi_{2}=0, \quad \mathrm{~d} \phi_{2}>0, \quad \text { or } \quad \hat{\tau}_{\mathrm{n}}{ }^{2}=\tau_{\mathrm{n}}{ }^{2}(\mathrm{t}), \quad \hat{\tau}_{\mathrm{n}} \mathrm{d} \hat{\tau}_{\mathrm{n}}=\tau_{\mathrm{n}} \mathrm{d} \tau_{\mathrm{n}}>0
\end{aligned}
$$

Figure $4 \mathrm{~b}$ presents four domains of loading-unloading specified by the corner regime at $P$. The non-local measure of increment of damage function is specified as follows

$$
d \overline{\hat{R}}_{\sigma}=\frac{1}{d_{0}^{2}} \int_{0}^{d_{0}} \int_{0}^{d} d \hat{R}_{\sigma} d \xi_{1} d \xi_{2}
$$

Let us apply the damage accumulation model to study crack initiation and damage distribution in the case of cyclic loading of a cylinder under combined bending and torsion. Assuming that $\sigma_{o} / \sigma_{c}=\tau_{o} / \tau_{c}=f$ and neglecting the effect of damage on values of $\sigma_{c}$ and $\tau_{c}$, the crack initiation condition is assumed in the form

$$
\mathbf{R}_{\mathrm{d}}=\max _{\left(\underline{\underline{n}}, \underline{x}_{o}\right)} \omega_{n \sigma}=\max _{\left(\underline{\mathbf{n}}, \underline{x}_{\sigma}\right.}\left[\int_{0}^{\hat{\mathrm{R}}_{\mathrm{d}}} \mathrm{A}_{\sigma}^{*}\left(\frac{\mathrm{R}_{\sigma}-\mathrm{f}}{1-\mathrm{f}}\right)^{\mathrm{n}_{\sigma}^{*}} \frac{\mathrm{d} \hat{\mathrm{R}}_{\sigma}}{1-\mathrm{f}}\right]=1
$$

Geometrically, for any stress point $P$ within the domain $\Omega^{*}$, the radial distance of this point from the curve $R_{\sigma o}=1$ equals $P_{0}$ and the radial distance between the curves $R_{\sigma}=1$ and $R_{\sigma o}=1$ equals $P_{c} P_{0}$. We have then

$$
\left(\frac{\mathrm{R}_{\sigma}-\mathrm{f}}{1-\mathrm{f}}\right)^{\mathrm{n}_{\sigma}^{*}}=\left(\frac{\mathrm{PP}_{\mathrm{o}}}{\mathrm{P}_{\mathrm{c}} \mathrm{P}_{\mathrm{o}}}\right)^{\mathrm{n}_{\sigma}^{*}}
$$

Consider a cylindrical element under cyclic loading by the bending and torsional moments, Figure 5. The resulting stress components are the axial stress $\sigma_{\mathrm{z}}$ and the shear stress $\tau_{\mathrm{r} \theta}$. The maximal stress

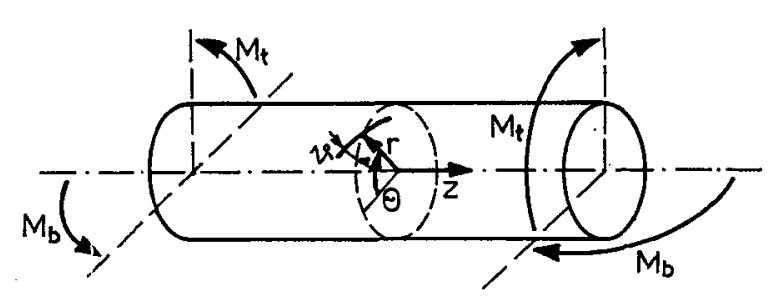

Figure 5. Cylindrical element and coordinate system $(\theta, r, z)$. values occur on the cylindrical surface and can be presented as follows

$$
\begin{aligned}
& \sigma_{z}(t)=\sigma_{a} \sin \omega t+\sigma_{m} \\
& \tau_{z \theta}(t)=\tau_{a} \sin (\omega t-\delta)+\tau_{m}
\end{aligned}
$$

where $\sigma_{\mathrm{a}}, \tau_{\mathrm{a}}$ are the normal and shear stress amplitudes, $\sigma_{\mathrm{m}}, \tau_{\mathrm{m}}$ denote their mean values, and $\delta$ is the phase-angle of two stress components. The stress components acting on any plane normal to the cylindrical surface are 


$$
\begin{aligned}
& \sigma_{\mathrm{n}}=\sigma_{\mathrm{no}}+\mathrm{k}_{\mathrm{o}} \cos (2 \vartheta+2 \beta) \\
& \tau_{\mathrm{n}}=\mathrm{k}_{\mathrm{o}} \sin (2 \vartheta+2 \beta)
\end{aligned}
$$

where

$$
\sigma_{\mathrm{no}}=\frac{1}{2} \sigma_{\mathrm{z}}, \quad \mathrm{k}_{\mathrm{o}}=\left[\left(\frac{\sigma_{\mathrm{z}}}{2}\right)^{2}+\tau_{\mathrm{z} \theta}{ }^{2}\right]^{1 / 2}
$$

and $\vartheta$ denotes the angle of orientation of the plane with respect to circumferential direction, $\beta$ denotes the orientation of the major principial stress plane. When the reference axes coincide with the principial axes, we set $\beta=0$ and then and then $\sigma_{n}=\sigma_{n o}+k_{0} \cos 2 \vartheta, \tau_{n}=k_{0} \sin 2 \vartheta$.
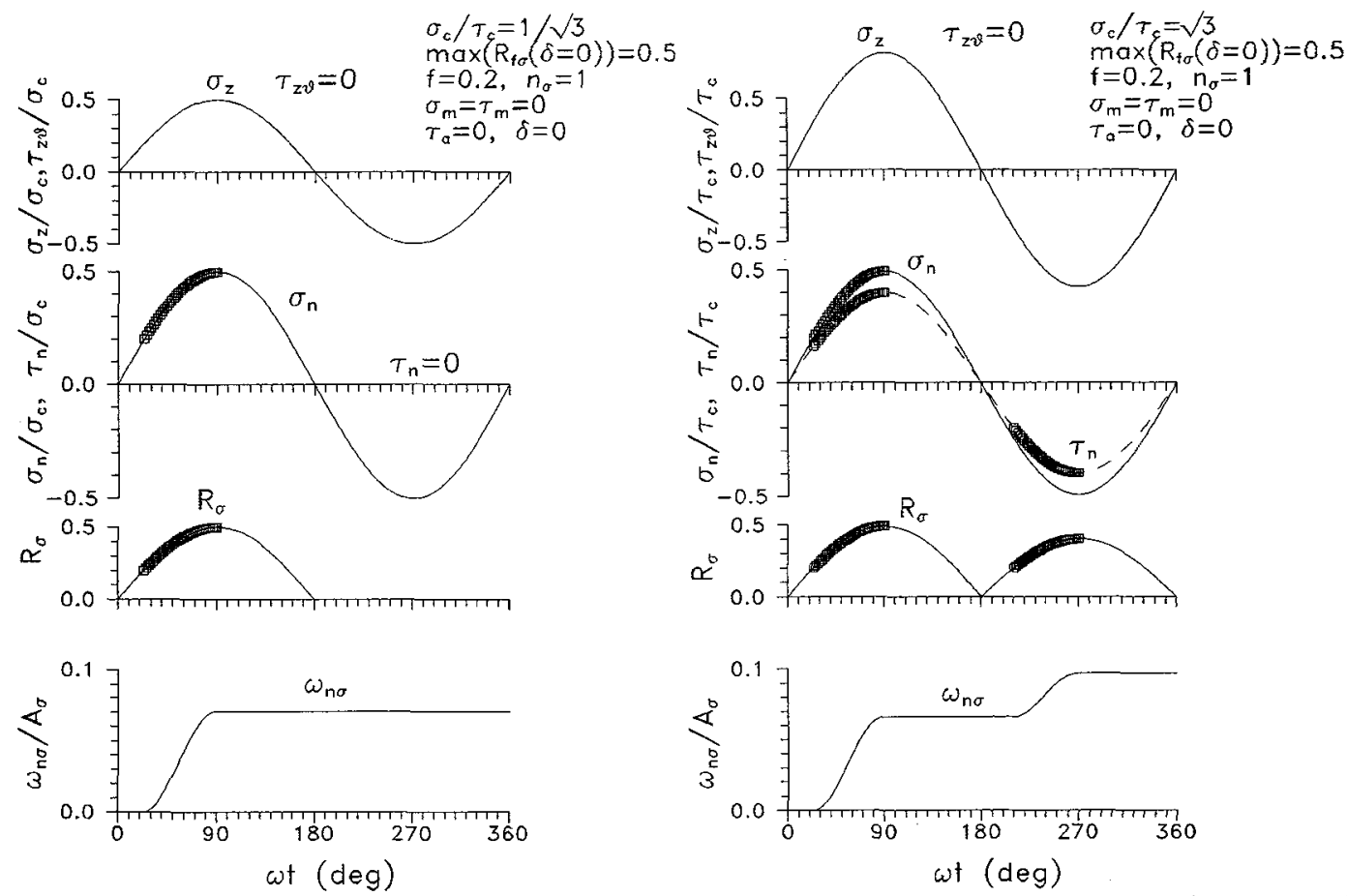

Figure 6. Damage accumulation on the extremal plane during one cycle of bending loading for: a) $\left.\tau_{c} / \sigma_{c}=1 / \sqrt{ } 3, b\right) \tau_{c} / \sigma_{c}=\sqrt{ } 3$,

Figures $6 a, b$ present the damage accumulation on the extremal plane during one cycle of in-phase loading $(\delta=0)$ and for the stress amplitude corresponding to $R_{f \sigma}=0.5$. It is assumed that $n_{\sigma}=1, f=0.2$, and the bending loading only, $\tau_{\mathrm{a}}=\tau_{\mathrm{m}}=0, \sigma_{\mathrm{m}}=0$. Figure $6 \mathrm{a}$ corresponds to $\sigma_{\mathrm{c}} / \tau_{\mathrm{c}}=1 / \sqrt{3}$ and Figure $6 \mathrm{~b}$ to $\sigma_{c} / \tau_{c}=\sqrt{3}$. The portions of stress cycle for wich damage accumulation occurs are marked as thickened segments. It is seen that damage occurs on different portions of the stress cycle and $\omega_{\text {no }}$ attains different values.

Figures $7 \mathrm{a}, \mathrm{b}$ present the damage accumulation diagrams for the out-of phase combined loading, $\tau_{\mathrm{m}}=\sigma_{\mathrm{m}}=0, \tau_{\mathrm{a}}=\sigma_{\mathrm{a}}, \delta=\pi / 2$ and for two values of $\sigma_{\mathrm{c}} / \tau_{\mathrm{c}}$ as in previous diagrams.

Figure 8 (reffering to the same case as Figure 6) presents rosette diagrams of damage parameter distribution on physical planes inclined at the angle $\vartheta$ to the plane normal to cylinder axis, for the bending cycles and for three values of mean and amplitude stress, namely $m=-1, m=-0.5$ and $m=0$. It is seen that the maximal damage plane orientation is essentialy affected by the values of $\mathrm{m}$. Figure 9 presents similar diagrams for non-proportional loading, and refers the case of loading illustrated in Figure 7. 

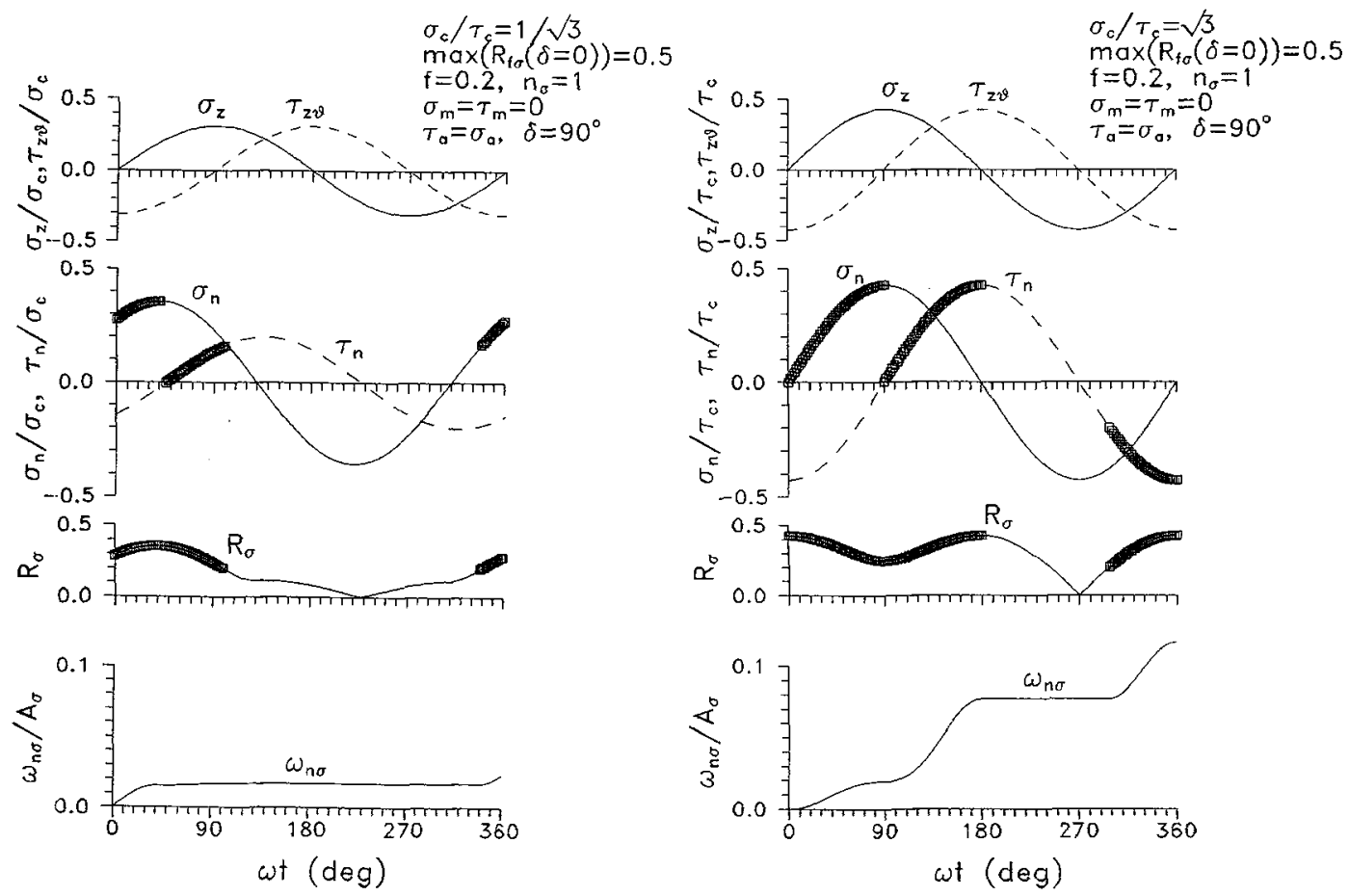

Figure 7. Damage accumulation on the extremal plane during one cycle of out-of-phase bending and torsional loading for: a) $\left.\tau_{\mathrm{c}} / \sigma_{\mathrm{c}}=1 / \sqrt{3}, \mathrm{~b}\right) \tau_{\mathrm{c}} / \sigma_{\mathrm{c}}=\sqrt{3}$,
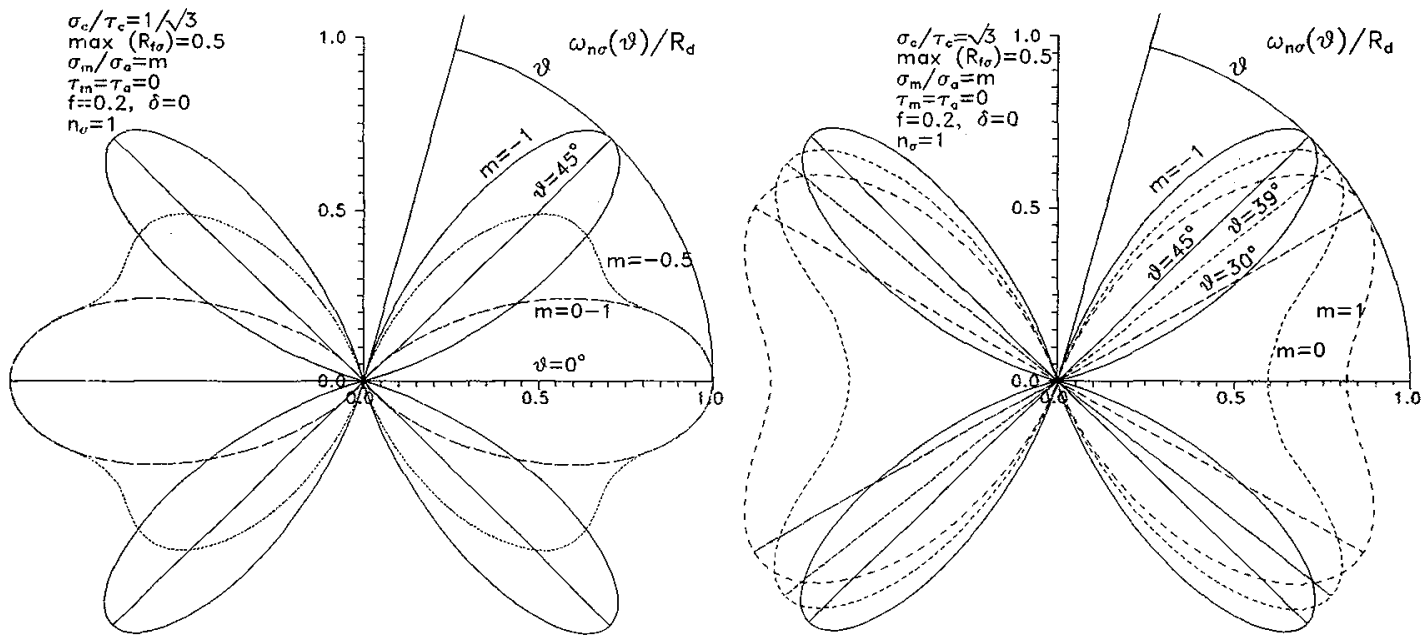

Figure 8. Rosette diagrams of damage distribution for the bending loading: a) $\left.\tau_{c} / \sigma_{c}=1 / \sqrt{3}, b\right) \tau_{c} / \sigma_{c}=\sqrt{3}$, 

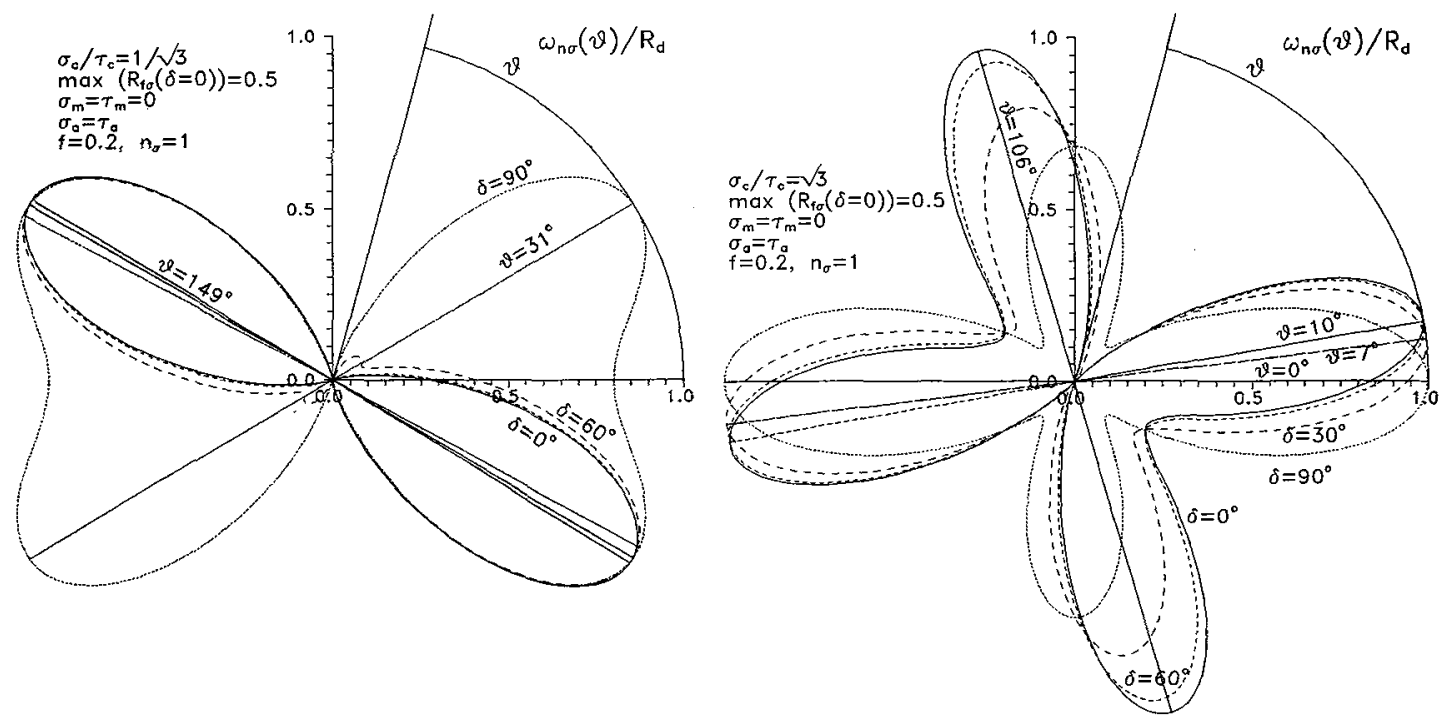

Figure 9. Rosette diagrams of damage distribution for the out-of-phase bending and torsional loading: a) $\tau_{\mathrm{c}} / \sigma_{\mathrm{c}}=1 / \sqrt{3}$, b) $\tau_{\mathrm{c}} / \sigma_{\mathrm{c}}=\sqrt{3}$,

\section{DAMAGE TENSOR REPRESENTATION}

The scalar damage distribution on physical planes predicted by the present model can be used in order to construct the tensor state variable of damage. In fact, the function $\omega_{n}(\underline{n})$ can be generated for any loading program. The mean value of damage within the element equals

$$
\omega_{0}=\frac{1}{4 \pi} \int_{4 \pi} \omega_{\mathrm{n}}(\underline{\mathrm{n}}) \mathrm{d} \Omega
$$

and the maximal value

$$
\omega_{\mathrm{d}}=\max _{(\underline{n})} \omega_{\mathrm{n}}(\underline{\mathrm{n}})
$$

is used to specify the crack initiation condition. Folowing the previous work of Kanatani [9] and Lubarda and Krajcinovic [10], the use of second and fourth-order damage tensors will be discussed.

For a second order damage tensor $\omega_{\mathrm{ij}}$, the damage measure on the physical plane equals

$$
\omega_{\mathrm{n}}(\underline{\mathrm{n}})=\omega_{\mathrm{ij}} \mathrm{n}_{\mathbf{i}} \mathrm{n}_{\mathbf{j}}
$$

Consider a tensor $\omega_{\mathrm{ij}}^{*}$ obtained from the known distribution $\omega_{\mathrm{n}}(\underline{\mathrm{n}})$, namely

$$
\omega_{i j}^{*}=\frac{3}{4 \pi} \int_{4 \pi} \omega_{n}(\underline{n}) n_{i} n_{j} d \Omega
$$

It is easy to show that the tensors $\omega_{\mathrm{ij}}$ and $\omega_{\mathrm{ij}}^{*}$ are interrelated by the equation

$$
\omega_{\mathrm{ij}}=\frac{5}{2} \omega_{\mathrm{ij}}^{*}-\frac{3}{2} \delta_{\mathrm{ij}} \omega_{\mathrm{o}}
$$

Thus, the damage tensor $\omega_{\mathrm{ij}}$ can be identified in terms of $\omega_{\mathrm{ij}}^{*}$ and $\omega_{\mathrm{o}}$ specified from the known distribution of scalar damage on physical planes. 

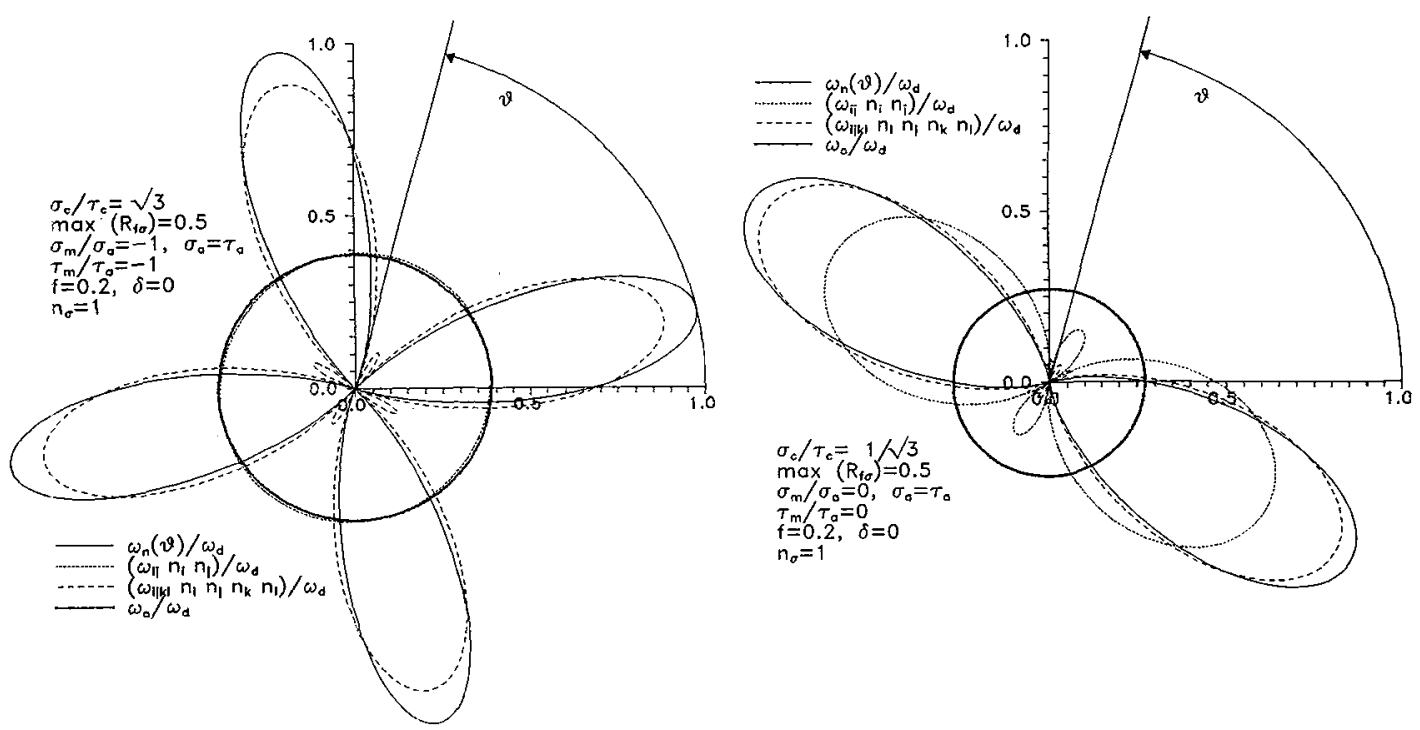

Figure 10. Rosette diagrams of damage distribution $\omega_{n}(\vartheta)$ and approximations by the second and fourth order tensors

When the fourth order damage tensor $\omega_{\mathrm{ijk}}$ is applied, the damage measure on the plane equals

$$
\omega_{\mathfrak{n}}(\underline{\mathrm{n}})=\omega_{\mathrm{ijkl}} \mathrm{n}_{\mathrm{i}} \mathrm{n}_{\mathrm{j}} \mathrm{n}_{\mathrm{k}} \mathrm{n}_{1}
$$

On the other hand, knowing $\omega_{\mathrm{n}}(\underline{\mathrm{n}})$, the auxiliary damage tensor $\omega_{\mathrm{ijkl}}^{*}$ can be obtained, namely

$$
\omega_{\mathrm{ijkl}}^{*}=\frac{5}{4 \pi} \int_{4 \pi} \omega_{\mathrm{n}}(\underline{\mathrm{n}}) \mathrm{n}_{\mathrm{i}} \mathrm{n}_{\mathrm{j}} \mathrm{n}_{\mathrm{k}} \mathrm{n}_{1} \mathrm{~d} \Omega
$$

The tensors $\omega_{\mathrm{ijkl}}$ and $\omega_{\mathrm{ijkl}}^{*}$ are now interrelated by the following equation

where

$$
\omega_{\mathrm{ijkl}}=\frac{63}{8} \omega_{\mathrm{ijkl}}^{*}-\frac{35}{4} \mathrm{~A}_{\mathrm{ijkl}}^{*}+\frac{15}{8} \mathrm{I}_{\mathrm{ijk} \mathrm{k}} \omega_{\text {。 }}
$$

$$
\begin{aligned}
& \mathrm{A}_{\mathrm{ijkl}}^{*}=\frac{1}{6}\left(\delta_{\mathrm{ij}} \omega_{\mathrm{kl}}^{*}+\delta_{\mathrm{kl}} \omega_{\mathrm{ij}}^{*}+\delta_{\mathrm{ik}} \omega_{\mathrm{jl}}^{*}+\delta_{\mathrm{il}} \omega_{\mathrm{jk}}^{*}+\delta_{\mathrm{jk}} \omega_{\mathrm{il}}^{*}+\delta_{\mathrm{jl}} \omega_{\mathrm{ik}}^{*}\right) \\
& \mathrm{I}_{\mathrm{ijkl}}=\frac{1}{3}\left(\delta_{\mathrm{ij}} \delta_{\mathrm{kl}}+\delta_{\mathrm{ik}} \delta_{\mathrm{jl}}+\delta_{\mathrm{il}} \delta_{\mathrm{jk}}\right)
\end{aligned}
$$

The relation (35) provides the damage tensor $\omega_{\mathrm{ijk} l}$ in terms of the specified distribution $\omega_{\mathrm{n}}(\underline{\mathrm{n}})$. For the plane case, the respective formulae are

$$
\begin{aligned}
& \omega_{\mathrm{o}}=\frac{1}{2 \pi} \int_{2 \pi} \omega_{\mathrm{n}}(\vartheta) \mathrm{d} \vartheta \\
& \omega_{\mathrm{ij}}^{*}=\frac{1}{\pi} \int_{2 \pi} \omega_{\mathrm{n}}(\vartheta) \mathrm{n}_{\mathrm{i}} \mathrm{n}_{\mathrm{j}} \mathrm{d} \vartheta \\
& \omega_{\mathrm{ijkl}}^{*}=\frac{4}{3 \pi} \int_{2 \pi} \omega_{\mathrm{n}}(\vartheta) \mathrm{n}_{\mathrm{i}} \mathrm{n}_{\mathrm{j}} \mathrm{n}_{\mathrm{k}} \mathrm{n}_{1} \mathrm{~d} \vartheta
\end{aligned}
$$

and the damage tensors $\omega_{\mathrm{ij}}$ and $\omega_{\mathrm{ijkl}}$ are expressed as follows 


$$
\begin{aligned}
& \omega_{\mathrm{ij}}=2 \omega_{\mathrm{ij}}^{*}-\delta_{\mathrm{ij}} \omega_{\mathrm{o}} \\
& \omega_{\mathrm{ijkl}}=6 \omega_{i \mathrm{jkl}}^{*}-6 \mathrm{~A}_{\mathrm{ijkl}}^{*}+\mathrm{I}_{\mathrm{ijk} \mathrm{l}} \omega_{\mathrm{o}}
\end{aligned}
$$

Figures $10 a, b$ present the description of damage distribution by the second and fourth order damage tensors for the cases loading discussed in the previous section, cf. Figures 6-9. It is seen that the second order damage tensor cannot describe adequately the damage distribution on physical planes. Much better description is provided by the fourth-order tensor.

The elastic compliance variation due to damage can be derived similarly assuming normal and tangential compliance on each material plane to be specified by the local damage $\omega_{n}(\underline{n})$.

\section{CONCLUDING REMARKS}

The damage and fracture conditions were expressed in terms of interface stresses or strains. The damage distribution within the element can then be determined and the crack initiation is assumed to occur within the plane of maximal damage. The singular and regular stress regimes can be uniformly treated by applying the non-local condition. The damage distribution function can be used to specify the damage tensor and compliance variation.

\section{References}

[1] Kachanov L.M., On the creep rupture time, Izv.A.N. SSSR, Otd. Techn. Nauk, 8 (1958), 26-31.

[2] Lemaitre J., A Course on Damage Mechanics, Springer Verl., Berlin (1992).

[3] Dragon A. and Mróz Z., A continuum model for plastic-brittle behaviour of rock and concrete, Int. J. Eng. Sci., 17 (1979), 121-137.

[4] Krajcinowic D., Damage mechanics, Mech.Mater., 8 (1989), 117-197.

[5] Murakami S., Mechanical modelling of material damage, J.Appl.Mech., 55 (1988), 280-286.

[6] Onat E.T., and Leckie F.A., Representation of mechanical behaviour in the presence of changing internal structure, J.Appl.Mech., 55 (1988), 1-10.

[7] Simo J.C. and Ju J.W., Strain and'stress based continuum damage models - I. Formulation, Int. J. Solids Struct, 23 (1987), 821-840.

[8] Chaboche J.L., Damage induced anisotropy: on he difficulties associated with the active/pasive unilateral condition, Int. J.Damage Mech., 1 (1992), 148-171

[9] Kanatani K., Distribution of directional data and fabric tensors, Int. J. Engng Sci., 22 (1984), 149-164.

[10] Lubarda, V.A., and Krajcinowic, D. , Damage tensors and the crack density distribution, Int. J. Solids Struct., 30 (1993), 2859-2877.

[11] Seweryn A., Brittle fracture criterion for structures with sharp notches, Engng Fract. Mech., 47 (1994), 673-681.

[12] Seweryn A. and Mróz Z., A non-local stress failure condition for structural elements under multiaxial loading, Engng Fract. Mech., 51 (1995), 955-973.

[13] Seweryn A. and Mróz Z., A non-local mixed mode fracture initiation and propagation condition for monotonic and cyclic loading. In: Proc. Fourth Int. Conf.on Biaxial/Multiaxial Fatigue, St Germain en Laye, 1 (1994), 499-512.

[14] Seweryn A and Mróz Z., A non-local stress failure and fatigue damage accumulation condition. In: Multiaxial Fatigue and Design (eds. Pineau A., Cailletaud G. and Lindley T.), Mechanical Engineering Publications (1996), 259-280. 